\section{IDDF2021-ABS-0201 STUDY ON THE PROMOTION OF LIPOPOLYSACCHARIDE FROM SHEWANELLA ALGAE ON THE OCCURRENCE AND DEVELOPMENT OF COLORECTAL ADENOMA}

Junxiao Zhou*, Junyan Li, Kaijie Liu, Yanxiao Liang, Hong Wang. Department of Gastroenterology and Hepatology, Guangzhou Digestive Disease Center, Guangzhou First People's Hospital, School of Medicine, South China University of Technology, Guangzhou, China

\subsection{6/gutjnl-2021-IDDF.59}

Background Colorectal adenoma (CRA) is a recognized precancerous lesion of colorectal cancer (CRC). Intestinal flora is closely related to many diseases, including CRA and CRC. Our previous study found that the abundance of Shewanella algae (S.algae) was significantly increased in CRA patients. Next, it was found that S.algae can significantly promote the development of CRA in $\mathrm{APC}^{\mathrm{min} /+}$ mice, and the levels of macrophages and lipopolysaccharide (LPS) were increased after using S.algae to gavage $\mathrm{APC}^{\mathrm{min} /+}$ mice. The bioactivity of LPS from different bacteria is different. Escherichia coli (E.coli) LPS has been shown to promote the development of tumors, while the bioactivity of S.algae LPS to CRA is not yet clear.

Methods Twenty-four female C57BL/6J mice were randomly divided into the Control group, the AOM/DSS group, the $\mathrm{AOM} / \mathrm{DSS}+\mathrm{LPS}$ group, and the AOM/DSS+S.algae group, with 6 mice in each group. The mice in the three experimental groups were given an intraperitoneal injection of $\mathrm{AOM}$ $10 \mathrm{mg} / \mathrm{kg}$, while the Control group was injected with the same amount of normal saline. After a week, the mice in the three experimental groups were regrouped according to body weight and given $2.5 \%$ DSS solution for 5 days and normal drinking water for 14 days. The mice were weighed every 3 days and killed at the end of the experiment on the 26th day. S.algae and its LPS were administered began on the eighth day and was gavaged every two days until the end of the experiment, while the Control group were given an equal amount of PBS by gavage.

Results Compared with the AOM/DSS group, mice in the AOM/DSS+LPS group and the AOM/DSS+S.algae group developed more intestinal polyps (IDDF2021-ABS-0201 Figure 1. Body mass, colorectal length, and polyps of mice in each group) and more severe atypical hyperplasia (IDDF2021-ABS0201 Figure 2. The pathological changes of the colorectum in mice of each group). M1 macrophages (CD11b+/CD86+)


AOM/DSS

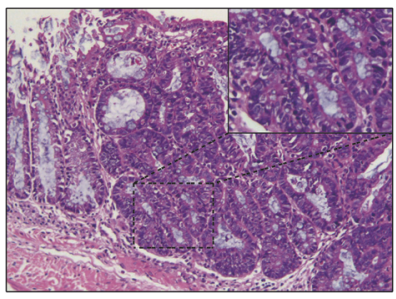

AOM/DSS+LPS

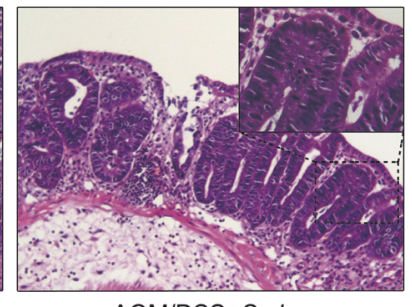

AOM/DSS+S.algae
Abstract IDDF2021-ABS-0201 Figure 2
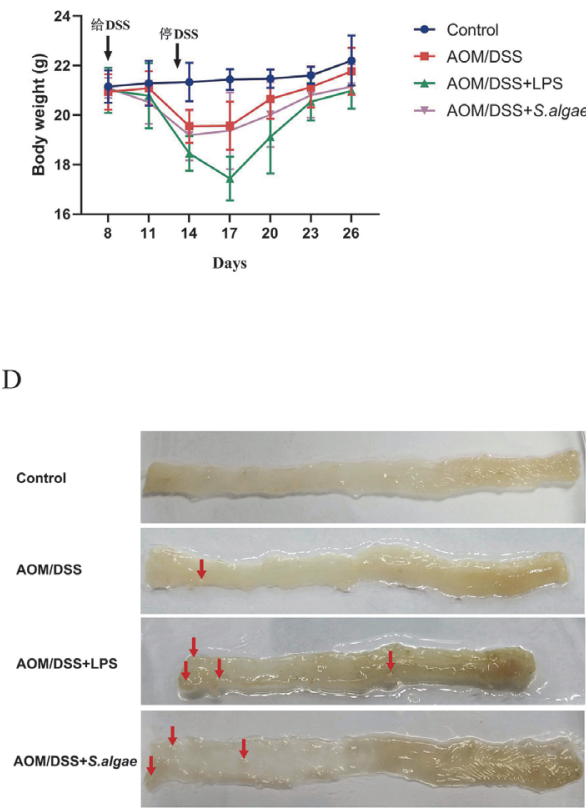

B

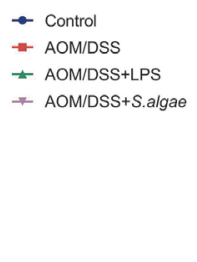

$\rightarrow$ AOM/DS

$\neq$ AOM/DSS+LPS

* AOM/DSS + S.algae

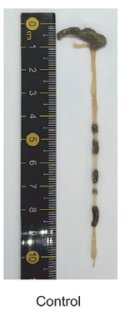

E
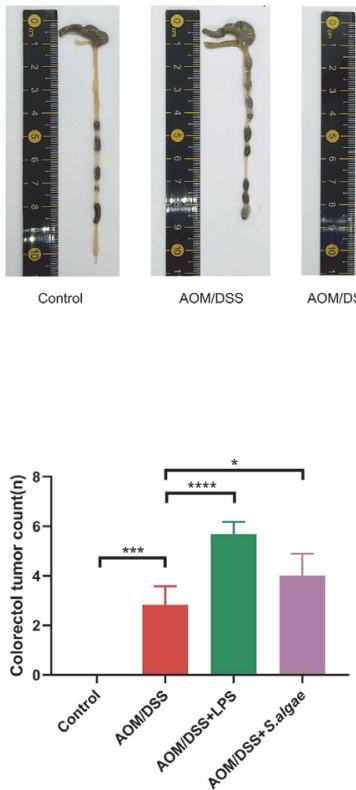

C
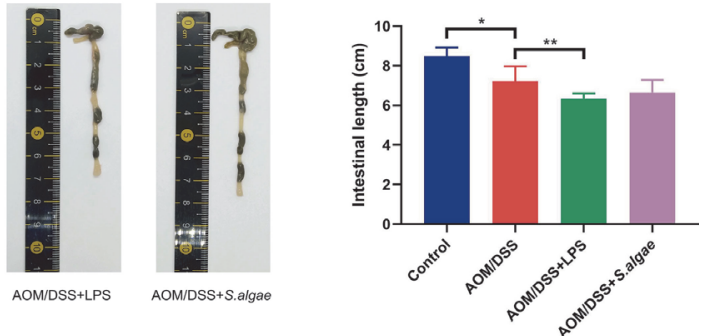

F 
A


B

E
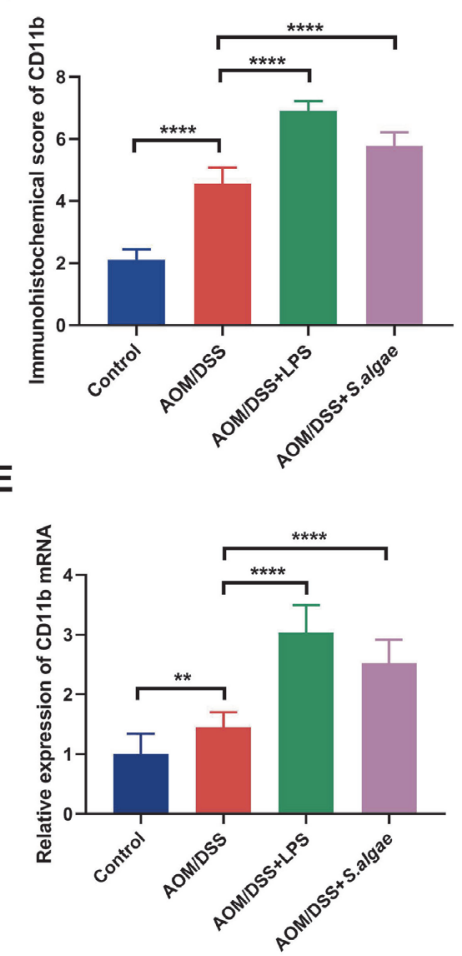

AOM/DSS
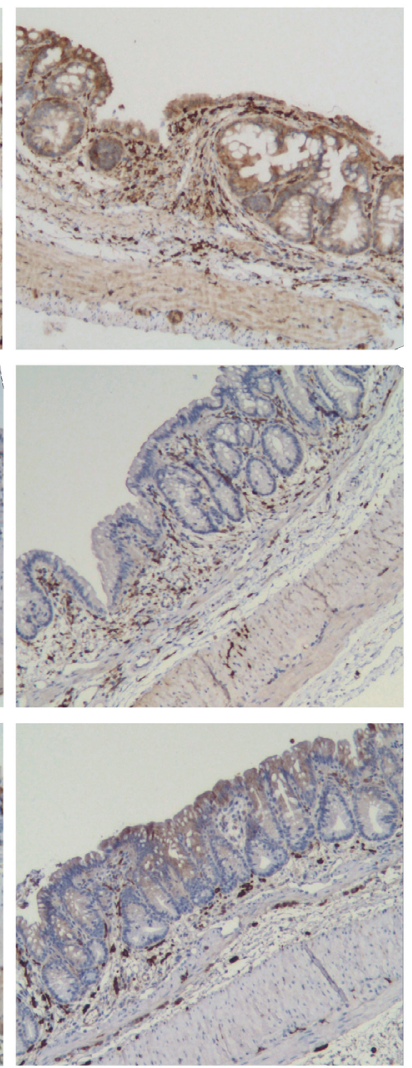

C

F
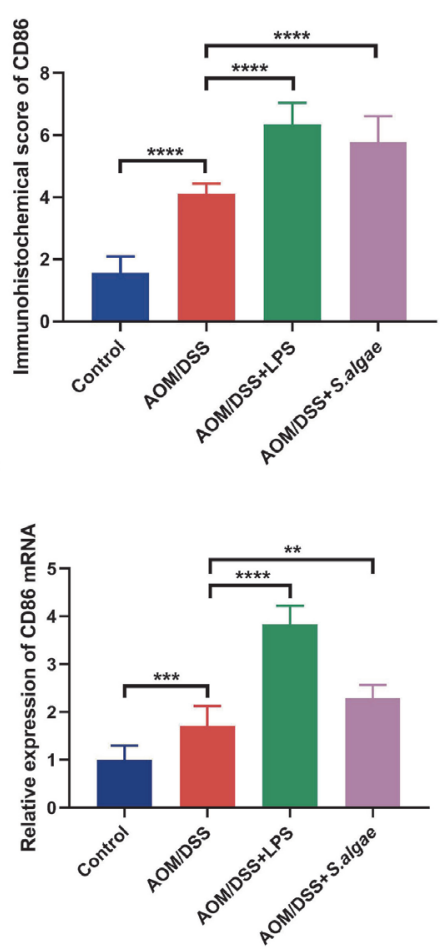

AOM/DSS+S.algae
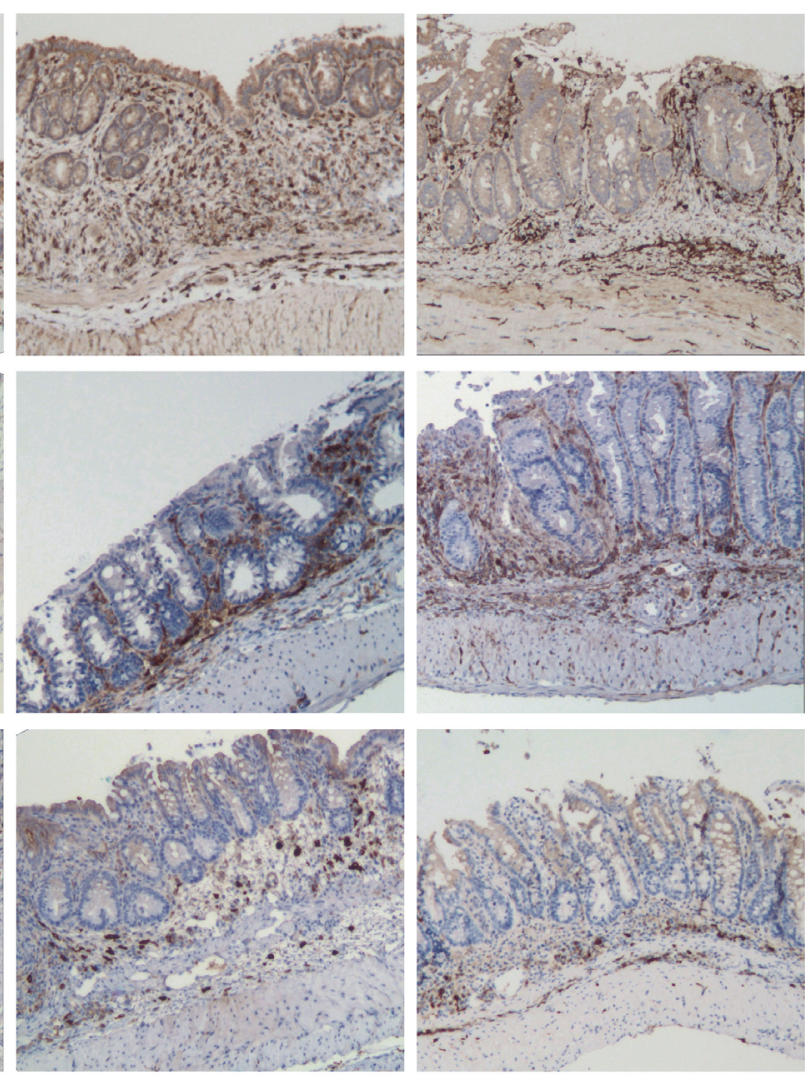

D

G
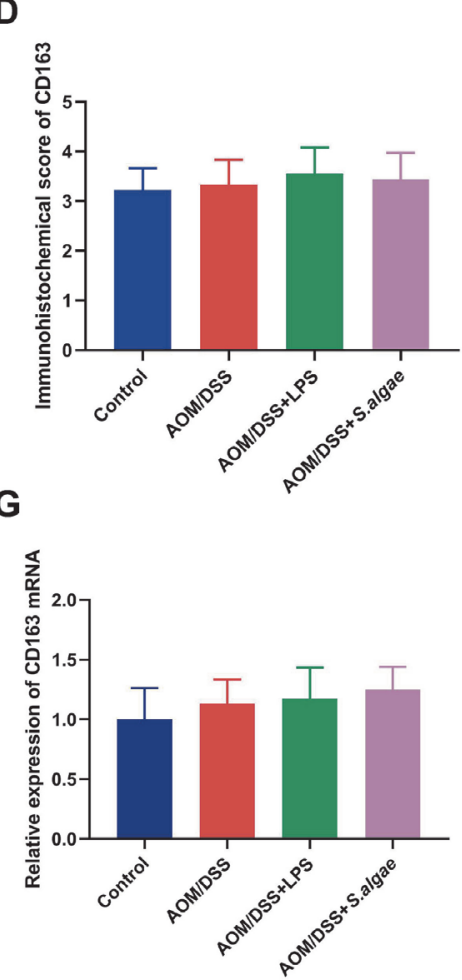

Abstract IDDF2021-ABS-0201 Figure 3 
A Control

AOM/DSS

AOM/DSS+LPS AOM/DSS+S.algae

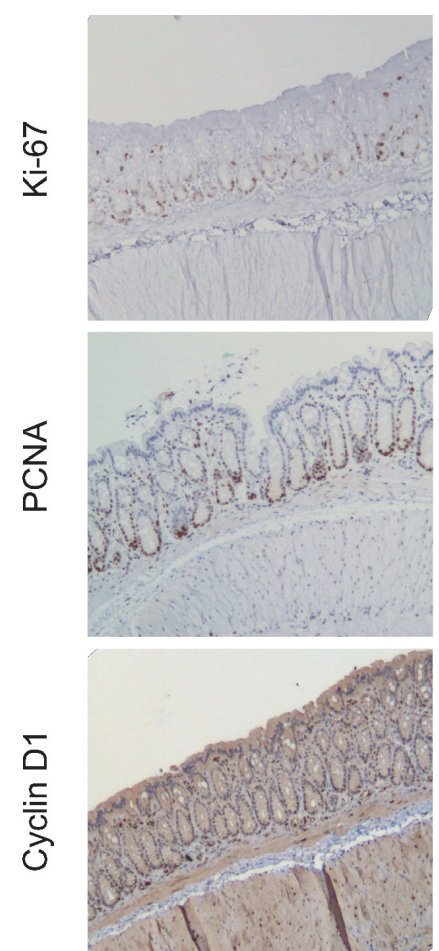

B

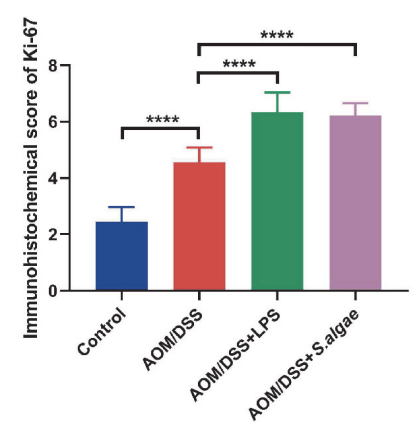

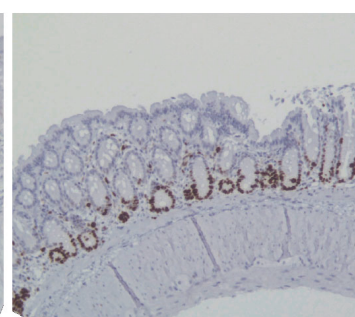
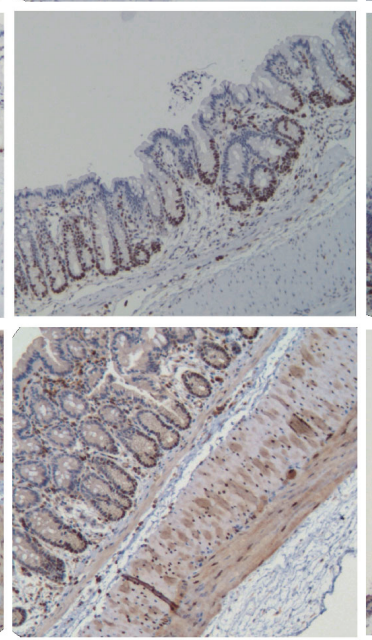

C

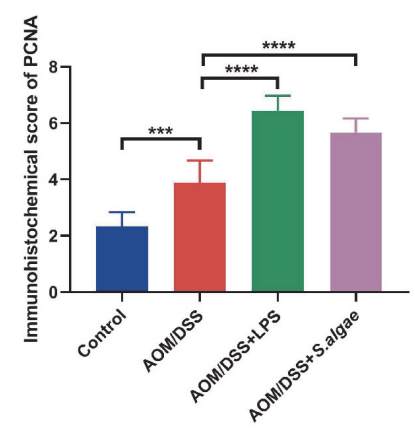


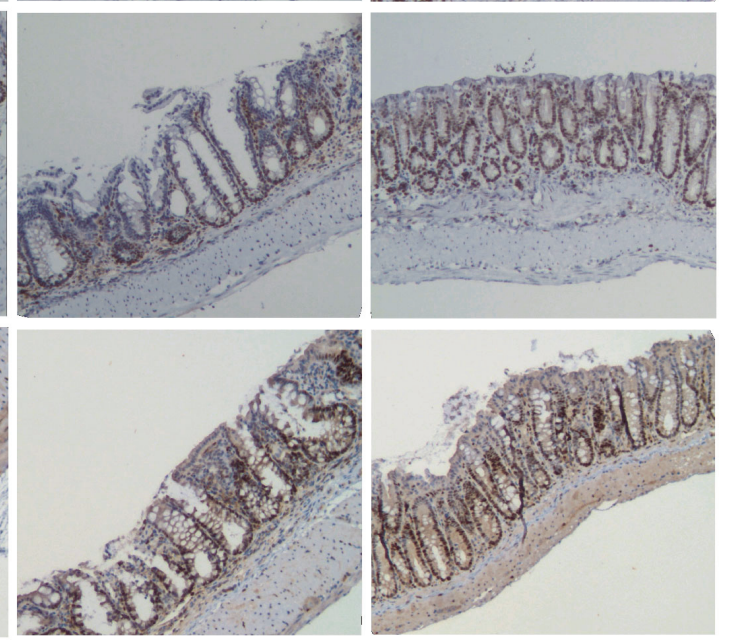

D


Abstract IDDF2021-ABS-0201 Figure 4
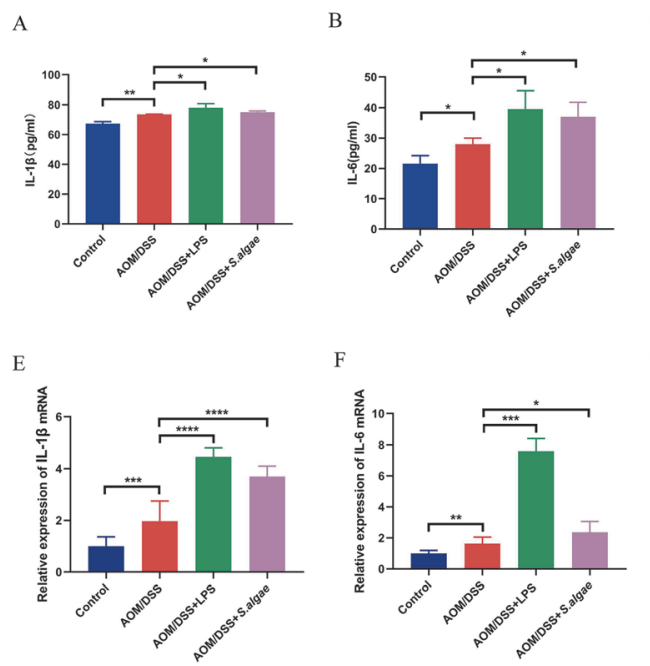

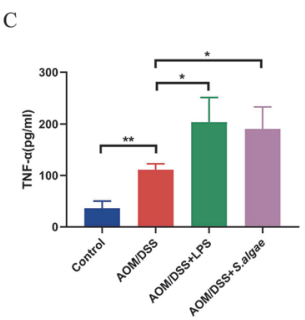

D

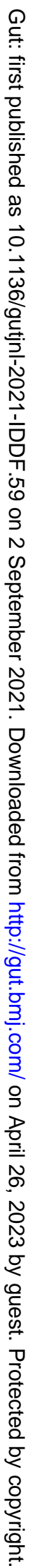

Abstract IDDF2021-ABS-0201 Figure 5


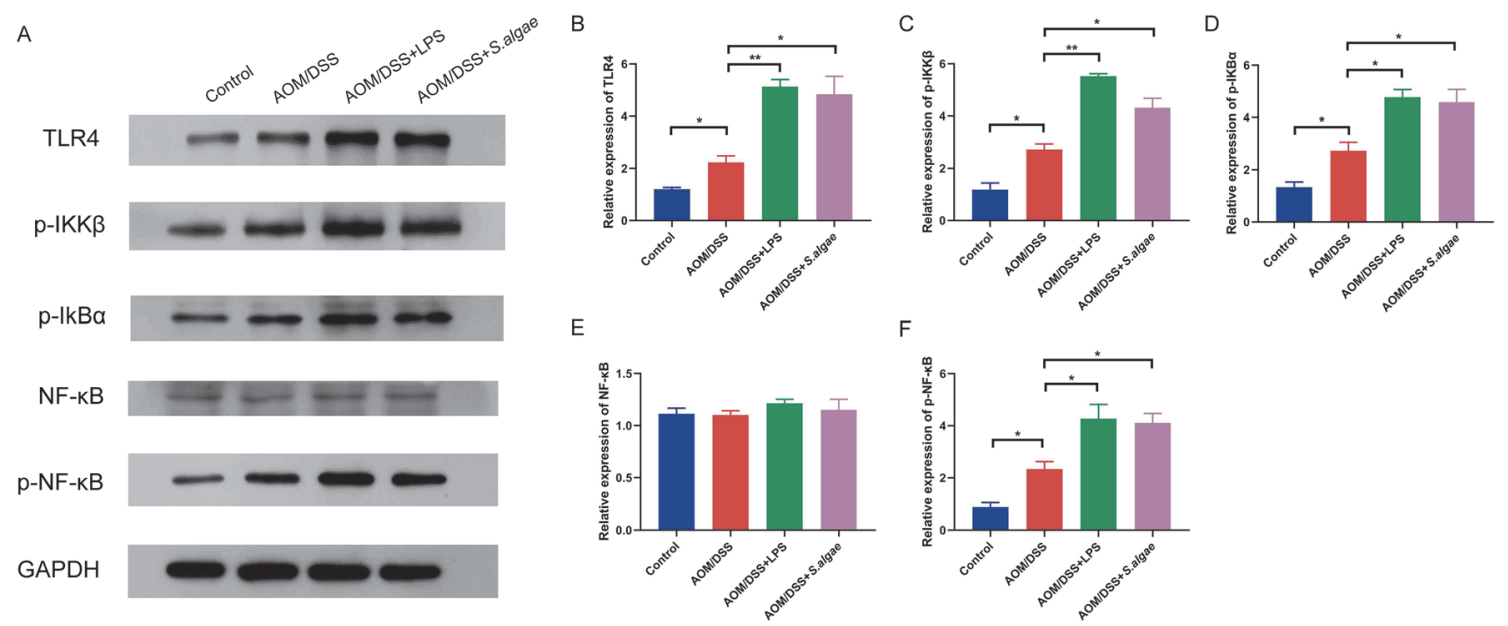

\section{Abstract IDDF2021-ABS-0201 Figure 6}

(IDDF2021-ABS-0201 Figure 3. The protein and mRNA levels of CD11b, CD86, CD163 in adenoma tissue of each group), proliferation and periodic proteins (Ki67, PCNA, Cyclin D1) (IDDF2021-ABS-0205 Figure 4.The protein levels of Ki-67, PCNA, and Cyclin D1 in adenoma tissue of each group) and various pro-inflammatory factors (IDDF2021-ABS-0201 Figure 5. The expression of protein and mRNA of inflammatory factors in adenoma tissue of each group) were elevated, and TLR4/NF-кB pathway (IDDF2021-ABS-0201 Figure 6. The expression of TLR4/NF- $\mathrm{KB}$ pathway proteins in adenomas tissue of mice in each group) was activated after using S.algae and its LPS to gavage mice.

Conclusions S.algae LPS may promote the occurrence and development of CRA in mice through M1 macrophages. S. algae LPS may promote the occurrence and development of CRA in mice through TLR4/NF- $\mathrm{B}$ pathway.

\section{IDDF2021-ABS-0212 FECAL MICROBIOTA TRANSPLANTATION AMELIORATES EXPERIMENTAL COLITIS BY REGULATING AUTOPHAGY}

Haoming $\mathrm{Xu}^{*}$, Diwen Shou, Yandi Liu, Jiaqi Zhu, Hongli Huang, Chong Zhao, Yao Peng, Youlian Zhou, Huiting Chen, Yuan Zhang, Yongjian Zhou, Yuqiang Nie. Department of Gastroenterology and Hepatology, Guangzhou Digestive Disease Center, Guangzhou First People's Hospital, School of Medicine, South China University of Technology, China

\subsection{6/gutjnl-2021-IDDF.60}

Background Autophagy is an important regulatory process to coordinate the homeostasis of intestinal epithelial cells under stress and mediate pathogen clearance. Fecal microbiota transplantation(FMT) is an important treatment for ulcerative colitis. In this study, we constructed autophagy activated/inhibited dextran sulfate sodium (DSS) induced colitis mice and observed whether FTM improve experimental colitis by regulating autophagy.

Methods Thirty male BALB/c mice were randomly divided into five groups: healthy control group (Control), DSS induced colitis group (DSS), autophagy activated colitis group (DSS + RAPA), autophagy inhibited colitis group (DSS +CQ) and autophagy inhibited and FMT intervention group (DSS $+\mathrm{CQ}+\mathrm{FMT}$ ). 3\% DSS drinking water was used to induce colitis, and the following interventions were given daily for seven days: Control group and DSS group were administered with PBS by gavage and intraperitoneal injection, DSS + RAPA group were administered with PBS by gavage and rapamycin $(4 \mathrm{mg} /$ $\mathrm{kg}$ ) by intraperitoneal injection, DSS +CQ group were administered with hydroxychloroquine $(40 \mathrm{mg} / \mathrm{kg})$ by gavage and PBS by intraperitoneal injection, DSS $+\mathrm{CQ}+\mathrm{FMT}$ group were administered with hydroxychloroquine $(40 \mathrm{mg} / \mathrm{kg})$ and PBS by intraperitoneal injection, and eight hours later administered FMT from healthy mice $(100 \mu \mathrm{L} / 10 \mathrm{~g})$ by gavage. Disease activity index (DAI) were monitored daily. On the eighth day, colonic tissues were resected after the mice were euthanized. The colon length was recorded, and some tissues were collected for histopathologic evaluation, while some tissues for observing autophagy expression (P62, LC3B and TFEB) by PCR

Results DAI $(\mathrm{P}=0.0128)$, colon length $(\mathrm{P}=0.043)$, and colon histopathologic score $(\mathrm{P}=0.0392)$ of colitis mice were effectively improved by autophagy activation (rapamycin), while DAI $(\mathrm{P}=0.4178)$, colon length $(\mathrm{P}=0.0393)$ and colon histopathologic score $(\mathrm{P}=0.765)$ were worsened markedly by autophagy inhibition (hydroxychloroquine); After autophagy inhibition, the following indexes of colitis were still significantly improved by FMT: DAI $(\mathrm{P}=0.0205)$, colon length $(\mathrm{P}=0.0008)$ and colon histopathologic score $(\mathrm{P}=0.0015)$ (IDDF2021-ABS-0212 Figure 1A. Fecal microbiota transplantation ameliorated experimental colitis and up-regulated autophagy expression, IDDF2021-ABS-0212 Figure 1B. Fecal microbiota transplantation ameliorated experimental colitis and up-regulated autophagy expression, IDDF2021-ABS0212 Figure 1C. Fecal microbiota transplantation ameliorated experimental colitis and up-regulated autophagy expression, IDDF2021-ABS-0212 Figure 1D. Fecal microbiota transplantation ameliorated experimental colitis and up-regulated autophagy expression), and the expression of the following autophagy genes were changed: P62 was significantly down-regulated $(\mathrm{P}=0.006)$, while LC3B and TFEB were significantly increased $(\mathrm{P}=0.0033 ; \quad \mathrm{P}=0.0039)$ (IDDF2021-ABS-0212 Figure 1E. Fecal microbiota transplantation ameliorated experimental colitis and up-regulated autophagy expression).

Conclusions Autophagy activation may be one of the mechanisms by which FMT improves DSS induced experimental colitis. 